
\title{
Reseacch S Suare \\ Comparative Analysis of Default Mode Networks in Major Psychiatric Disorders Using Resting-State EEG
}

\author{
Kang-Min Choi \\ Inje University \\ Jeong-Youn Kim \\ Korea Institute of Science and Technology (KIST) \\ Yong-Wook Kim \\ Inje University \\ Jung-Won Han \\ Inje University \\ Chang-Hwan Im ( $\nabla$ ich@hanyang.ac.kr) \\ Hanyang University \\ Seung-Hwan Lee \\ Inje University
}

\section{Research Article}

Keywords: Electroencephalography, default mode network, functional network, psychiatric disorders, clustering coefficient

Posted Date: June 30th, 2021

DOI: https://doi.org/10.21203/rs.3.rs-651053/v1

License: (c) (1) This work is licensed under a Creative Commons Attribution 4.0 International License.

Read Full License

Version of Record: A version of this preprint was published at Scientific Reports on November 10th, 2021. See the published version at https://doi.org/10.1038/s41598-021-00975-3. 


\section{Abstract}

Default mode network (DMN) is a set of functional brain structures coherently activated when individuals are in resting-state. In this study, we constructed multi-frequency band resting-state EEG-based DMN functional network models for major psychiatric disorders to easily compare their pathophysiological characteristics. Phase-locking values (PLVs) were evaluated to quantify functional connectivity; global and nodal clustering coefficients (CCs) were evaluated to quantify global and local activities of DMN nodes, respectively. DMNs of patients with post-traumatic stress disorder (PTSD), obsessive compulsive disorder (OCD), panic disorder, major depressive disorder (MDD), bipolar disorder, schizophrenia (SZ), mild cognitive impairment ( $\mathrm{MCl})$, and Alzheimer's disease (AD) were constructed relative to their demographically-matched healthy control groups. Overall DMN patterns were then visualized and compared with each other. In global CCs, SZ and AD showed hyperactivation in the theta band; OCD, $\mathrm{MCl}$, and AD showed hypoactivation in the low-alpha band; OCD and MDD showed hypoactivation and hyperactivation in low-beta, and high-beta bands, respectively. In local CCs, disease-specific patterns were observed. In the PLVs, lowered theta-band functional connectivity between the left lingual gyrus and the left hippocampus was frequently observed. Our comprehensive comparisons suggest EEG-based DMN as a useful vehicle for understanding altered brain networks of major psychiatric disorders.

\section{Introduction}

Default mode network (DMN) is a set of functional brain structures coherently activated when individuals are awake without engaging in any goal-directed activities ${ }^{1,2}$. With the increasing interest in so-called resting-state functional brain activity, studies of the resting-state DMN have been gradually paid attention to for several years ${ }^{3,4}$. Comparison of multiple psychiatric disorders is one of the promising research areas in functional networks. There are several advantages for the study of resting-state functional network of DMN because an individual does not need to be involved in specific tasks requiring attention, which could be easily affected by interrupting factors such as lack of attention due to low motivation or mind wandering ${ }^{5}$.

Relying on numerous advantages, DMN studies have been performed with a variety of modalities such as functional magnetic resonance imaging ( $\mathrm{fMRI}$ ), positron emission tomography (PET), and electroencephalography (EEG) ${ }^{6-10}$. Among them, EEG-based DMN is one of the competitive noninvasive and cost-effective modalities, which directly reflects brain electrical activity ${ }^{11,12}$. In addition, it can be analyzed into multiple frequency bands, allowing for diverse interpretations according to the characteristics of each frequency band. These beneficial properties help to possess potentials to become employed as a universal evaluation framework of psychiatric disease.

There have been a few resting-state EEG-based DMN studies to compare psychiatric disorders and healthy controls $(\mathrm{HC})$. For example, Hsiao et al. ${ }^{13}$ compared resting-state EEG between patients with mild cognitive disorder $(\mathrm{MCl})$ and Alzheimer's disease $(A D)$ in the $D M N$, showing various altered interconnections between them. Meanwhile, Krukow et al. ${ }^{14}$ observed theta-band DMN hyperconnectivity 
in first-episode schizophrenia (SZ) patients, suggesting that it might interfere with efficient cognitive function. Likewise, most of the EEG-based DMN studies have focused on the abnormalities of the specific disorder. However, these previous studies might be misleading or difficult to draw generalized pathophysiological characteristics of the DMN because of the lack of comprehensive comparison of a variety of psychiatric disorders, simultaneously with the same analytic methods.

In this exploratory study, we tried to construct EEG-based DMN functional network models based on a graph theory for a variety of major psychiatric disorder groups. We wanted to visualize the DMN patterns to readily compare each other, which comprised post-traumatic stress disorder (PTSD), obsessive compulsive disorder (OCD), panic disorder (PD), major depressive disorder (MDD), bipolar disorder (BD), $S Z, M C l$, and $A D$. We hypothesized that the $D M N$ patterns could easily contrast the characteristics of major psychiatric disorders, and they could reflect the pathophysiology of major psychiatric disorders.

\section{Methods And Materials}

\section{Participants}

A database in which patients were diagnosed as having psychiatric disorders from January 2006 to December 2018 from the Inje University Ilsan Paik Hospital was used. A diagnosis of these disorders was based on a clinical evaluation by trained psychiatrists using the Structured Clinical Interview for DSM-IV or V Axis I Disorders (SCID-I) or Mini international neuropsychiatric interview (MINI). Meanwhile, patients who were in pregnancy or possess other neurological or comorbid disorders, other organic brain damage, or impairment in sensory or motor function were excluded from the analysis. Finally, 104 SZ, 74 PTSD, 82 PD, 29 OCD, 69 MDD, 60 BD, $34 \mathrm{MCl}$, and 29 AD patients were included and analyzed in this study (see Supplementary Table S1 for detailed demographic information and see Supplementary Table S2 for medicine dosage information).

A total of 250 healthy participants were recruited from the local community using advertisements. They satisfied neither the DSM-IV nor V-based lifetime diagnostic criteria for any major psychiatric disorders, as screened by the SCID-I Non-Patient Edition (SCID-NP) nor MINI-based diagnostic criteria. For each disorder group, its corresponding $\mathrm{HC}$ participants were selected in pseudorandom for demographic information including age, sex, and education to be matched (Table S1).

The ethical approval was made by Inje University Ilsan Paik Hospital Institutional Review Board (IRB no. 2018-12-012-013). This study was conducted by retrospective data inspection without the prior written consent of specific study titles. However, the participants were explained that their EEG data could be used for research purposes and were required a written consent at the time of EEG measurement.

\section{Signal acquisition and pre-processing}

Resting-state EEG was recorded for 4 minutes in this study. The EEG signal was acquired using the SynAmps amplifier (Neuroscan, Compumedics USA, Charlotte, NC, USA) with $62 \mathrm{Ag} / \mathrm{AgCl}$ electrodes 
mounted on NeuroScan Quik-cap according to the international 10-10 system. The additional electrooculogram (EOG) signal was acquired with two electrodes each attached below the right eye and to the right of the outer canthus. The sampling rate of the equipment was set at 1,000 Hz. The impedance of each electrode was maintained below $5 \mathrm{k} \Omega$ during the whole experimental period. The ground and reference electrodes were placed on the forehead and both mastoids, respectively.

The acquired EEG data were manually inspected to eliminate segments contaminated by environmental or physiological noises. The eye movement artifact was removed using the independent component analysis. Subsequently, the signal was applied to common average reference and baseline correction and then segmented into $2 \mathrm{~s}$ epochs without an overlap. These pre-processing steps were performed using CURRY 7 software (Compumedics NeuroScan; Hamburg, Germany). Among the epoched data, 45 epochs were randomly selected and then further analyzed using Matlab R2019b (MathWorks; Natick, MA, USA). It is to be noted that the epochs with the maximum absolute value larger than $100 \mathrm{mV}$, were discarded.

\section{Construction of the DMN functional network model}

Regions of interest (ROI) The determination of the regions of interest (ROIs) and their coordinates was made based on 17 highly cited $(>500)$ articles that include coordinate information. Among a variety of candidate regions, those referred by more than three times regardless of the hemisphere were selected as the ROIs for DMN. Consequently, 25 DMN ROls were determined (Fig. 1a), including cingulate (Region 1 5; R1 5), frontal (R6 9), occipital (R10 13), parietal (R14 19), and temporal (R20 23) cortices, and hippocampus (R24 25). Each coordinate of the ROI was determined as the center of all coordinates for the candidate ROIs.

Source localization To calculate the source activities of ROIs from the recorded scalp EEG signals, a depth-weighted L2-norm estimator implemented in the Brainstorm toolbox ${ }^{36}$ was employed. The Colin27 MRI brain template and the deep brain structures provided by the Brainstorm toolbox were employed to estimate cortical activities and hippocampal activities, respectively. The lead field matrix was constructed using a three-layer boundary element model provided by the OpenMEEG project software ${ }^{37}$. Among the 30,020 nodes each with the estimated cortical current density values, those located within a $5 \mathrm{~mm}$ distance from the coordinates of each ROI were selected, and consequently, 25 clusters of nodes corresponding to $25 \mathrm{DMN}$ ROIs were constructed. The source signal of each ROI was then obtained by applying the principle component analysis to the source signals of all nodes in each ROI.

Network construction The DMN functional network model was constructed in accordance with a typical procedure described below: First, the source signals of $25 \mathrm{ROIs}$ (Fig. 1) were evaluated for each epoch as described in the previous paragraph. Second, the source signals of each ROI were decomposed into the following five frequency bands: theta $(4-8 \mathrm{~Hz})$, alpha $1(8-10 \mathrm{~Hz})$, alpha $2(10-12 \mathrm{~Hz})$, beta $1(12-$ $18 \mathrm{~Hz})$, and beta $2(18-30 \mathrm{~Hz})$. This signal decomposition was accomplished by using a $6^{\text {th }}$ order zerophase Butterworth infinite impulse response (IIR) band-pass filter implemented in the Matlab Signal Processing toolbox, with the cutoff frequencies equal to the borders of each frequency band. Third, the 
functional connectivity between every pair of the ROls was evaluated by the phase-locking value (PLV) that has been widely employed to evaluate phase synchronization ${ }^{38}$. The PLV between each ROI pair was evaluated by averaging PLVs of all 45 epochs for each patient. Fourth, after the functional connectivity network was constructed for each frequency band, the local clustering coefficient (CC) was evaluated to measure the local functional segregation status indicating a degree of nodal clustering within its neighboring nodes ${ }^{39}$. Finally, global CC was evaluated to quantify the overall clustering level of a network.

Visualization as DMN pattern The significance levels of the PLVs and local CCs were expressed by the thickness of the curve between a pair of nodes and the color intensity of the node region (Fig. 1). In the visualization of both properties, red (or blue) colors in the patterns indicated that the disease group shows significantly higher (or lower) values compared to its corresponding HC group.

\section{Statistical analysis}

All absolute values of skewness were lower than 2, and that of kurtosis was lower than 7 for every demographic distribution, satisfying the normality assumption of those distributions ${ }^{40}$. Two-tailed Student $t$ test was used to examine for age, sex, and education between comparing groups. To avoid multiple test issues, cluster-based permutation test $(n=10,000)$ was used to test the significance of PLV $(n=12,000)$ or $\mathrm{CC}(n=1,040)$ between pairing groups ${ }^{41}$.

\section{Results}

There were no significant differences between each pair of groups in terms of demographic characteristics, including age, sex, and education (Table S1). The functional network model of the DMN was constructed and then visualized based on the determined ROIs (Fig. 1). The significantly different local clustering coefficient (CC) activity and phase-locking value (PLV) connectivity of each disorder group are illustrated at a glance, compared with its demographically matched HC group (Fig. 2). The global CC activations are provided to compare major psychiatric disorders (Fig. 3). DMN patterns of 5 frequency bands were presented based on the frequency bands: theta, alpha1, alpha2, beta1, beta2.

\section{DMN patterns at a glance}

Several differences and similarities were observed in DMN patterns among the disease groups (Fig. 2). First, in CC activity, general homogenous activations were found in disease-specific manners. For example, $A D, M C l, S Z$, and $B D$ showed relative $C C$ hyperactivity of theta band compared to other disorders. MDD, PD, and PTSD showed relative CC hyperactivity of beta 2 band compared to other disorders. Second, in PLV connectivity, heterogeneous (mixed) patterns were found in disease-specific manners. For example, SZ and PTSD showed the high and low mixed regional connectivity patterns in theta and beta 2 frequency bands. These mixed connectivity patterns were also observed in beta1 band of MDD. 


\section{Disease-specific patterns of CC activities}

Globally, some disease groups showed significantly different global CC activation compared to their corresponding HC groups (Fig. 3; see Supplementary Table S3 for comparison of actual global CC values). In the theta band DMN, significantly higher global CC activations were observed in SZ $(p=0.017)$ and $A D(p=0.007)$ groups. In the alpha1 band, significantly lower global $C C$ activation was observed in $A D(p=0.036)$ group; in addition, marginally significantly lower global $C C$ activations were observed in $\mathrm{MCl}(\mathrm{p}=0.067)$ and OCD $(p=0.050)$ groups. In the beta1 band, significantly lower global CC activation was observed for OCD ( $p=0.022)$ group; while in the beta2 band, significantly higher global CC was observed for $\mathrm{MCl}(\mathrm{p}=0.031)$ group.

Locally, further analyses were performed with some DMN patterns showing aforementioned globally abnormal activation tendencies. In the theta band DMN, SZ and AD groups exhibited similar CC activation patterns (Fig. 4a): several regions were consistently hyperactive in both groups, particularly for left superior frontal gyrus ( $R 8, p=0.0034$ for both groups), left middle occipital gyrus ( $R 12, p=0.0072$ for $S Z ; p=0.0013$ for $A D)$, right precuneus $(R 17, p=0.0014$ for $S Z ; p=0.0045$ for $A D)$, and right superior temporal sulcus ( $R 23, p=0.0024$ for $S Z ; p=0.0019$ for $A D$ ). In the alpha 1 band, $O C D, M C l$, and $A D$ groups exhibited widespread lower local CC activation (Fig. 4b). Although each of them exhibited a common CC hypoactivation in left lingual gyrus ( $\mathrm{R} 10, p=0.0449$ for $\mathrm{MCl} ; \mathrm{p}=0.0100$ for $\mathrm{AD} ; \mathrm{p}=0.0033$ for OCD), the DMN patterns were quite different from each other. For Example, the OCD group showed significantly lower $\mathrm{CC}$ activation mainly in the posterior cingulate cortex (R3 5), whereas $\mathrm{MCl}$ and $\mathrm{AD}$ groups showed little difference in the regions. Meanwhile, the AD group showed widespread hypoactive regions compared to the $\mathrm{MCl}$ group. In the beta1 band, the OCD group showed strong CC hypoactivation in the right superior temporal sulcus ( $223, p=0.0030$; Fig. $4 \mathrm{c}$ ). In the beta2 band, the MDD group showed strong CC hyperactivation in the right lingual gyrus ( $R 11, p=0.0013$; Fig. $4 d)$. These high frequency band DMN patterns exhibited focal abnormal CC activation characteristics.

\section{Disease-specific patterns of PLV connectivities}

Interestingly, lowered functional connectivity of theta band between left lingual gyrus (R10: region number 10 in Fig. 1a and b) and left hippocampus (R24: region number 24 in Fig. 1a and b) was frequently observed over all disease (Fig. 5). However, statistically significant differences were found in the PTSD, BD, and SZ groups ( $<<0.001$ for BD and PTSD groups; $p<0.0001$ for SZ group), and in the PD and $\operatorname{MDD}(p=0.016$, and $p=0.030$, respectively $)$.

\section{Discussion}

In the present study, EEG-based DMN functional network models were constructed for 8 major psychiatric disorders based on a graph theory. The constructed DMNs were visualized, and then their DMN patterns were compared with each other. Overall DMNs patterns were well visualized at a glance. The diseasespecific global DMN patterns were discussed first and then several key local CC activities and PLV patterns were discussed. 


\section{DMN patterns at a glance}

DMN CC activity exhibited homogeneous alteration patterns according to their specific symptom. Disease groups known to show cognitive decline such as $A D, M C l, S Z$, and $B D^{14,15}$ exhibited relative $C C$ hyperactivity of theta band compared to the others (discussed in the following section). On the other hand, disease groups known to show anxiety symptoms exhibited relative CC hyperactivity of high frequency bands compared to the others. For example, PTSD, PD, and MDD showed relative CC hyperactivity of beta2 frequency band; OCD showed relative $\mathrm{CC}$ hyperactivity of alpha2 frequency band. These symptom-dependent alterations of the local CC activity in a specific frequency band are thought to be associated with particular pathophysiological symptoms (more detail in the following section).

Most PLV connectivity showed mixed patterns, which may imply distinct region-specific activation patterns, even in single frequency bands in a specific disease. These mixed patterns were dominantly observed in MDD and SZ. MDD and SZ are very heterogeneous disease entities among psychiatric disorders ${ }^{16,17}$. Even in single diagnostic entity, each patient could show a distinct activation pattern in DMN brain regions ${ }^{17}$. Our results agree with the previous studies reporting that MDD and SZ exhibited abnormal mixed functional patterns ${ }^{18,19}$.

\section{Disease-specific patterns of $\mathrm{CC}$ activities}

In the theta band $C C$, the $S Z$ and $A D$ groups showed predominant global hyperactivation. Our results agree with the previous reports in which dominantly hyperactive theta band DMN might reflect inefficient cognitive function ${ }^{20}$. Additional previous studies reported that whole-brain theta band functional connectivity increased in the patients with $S Z$ and $A D{ }^{20,21}$. In our study, both $S Z$ and $A D$ showed particularly higher activation for some common ROls, including the left superior frontal gyrus (R8), left middle occipital gyrus (R12), right precuneus (R17), and right superior temporal gyrus (R23). The theta band hyperactivation of fronto-temporo-patietal regions has been consistently reported in SZ and $A D^{20,21}$, which is linked to working and verbal memory. Theta hyperactivation of the occipital cortex might involve psychiatric symptoms such as dysfunction (i.e. illusion or hallucination) of the visual system ${ }^{22}$, which is commonly observed in both disorders. However, the AD group showed more widespread hyperactivation than the SZ group, implying more severe neuronal degeneration.

In the alpha1 band $\mathrm{CC}$, the $\mathrm{OCD}, \mathrm{MCl}$, and $\mathrm{AD}$ groups showed consistent global hypoactivation. In the previous studies, interregional alpha band synchrony is thought to be related to the coordinating functional integration ${ }^{23}$. The deterioration of functional integration can easily affect the cognitive decline. Therefore, our findings support that cognitive function decline is one of the consistent symptoms not only in patients with $\mathrm{MCl}$ and $A D$ but also in patients with $O C D^{21,24}$. Considering this significant deterioration was not observed in the alpha2 band, the integrational function might be more related to the low alpha (alpha 1) band synchrony. Locally, our study revealed that the left lingual gyrus (R10) was commonly hypoactive in all groups. The lingual gyrus is linked to the visual system ${ }^{25,26}$. Meanwhile, in the $\mathrm{MCl}$ group, the hypoactive regions include mainly the parietotemporal cortex, especially for the right 
middle temporal cortex (R21). However, in the AD group, the regions were more spread broadly including the fronto-occipital cortex and hippocampus. The regional broadening involved suggests a progression of neuronal degeneration from $\mathrm{MCl}$ to $\mathrm{AD}$. Generally, it is well known that structural or functional abnormality begins at the temporal lobe in the patients with early state $\mathrm{MCl}$, and then gradually broadened to other brain regions in patients with $A D^{25}$. In the OCD group, the main hypoactive region was the posterior cingulate cortex, distinctively from other disease groups. Previous works reported that the gray matter volume and resting-state metabolism of the posterior cingulate cortex consistently increased in OCD patients ${ }^{27,28}$, different from the other groups. Consequently, it can be presumed that resting-state functional hypoactivation of the posterior cingulate cortex in the OCD group is actually caused by excessive mental load such as obsessive rumination.

In the beta 1 band $C C$, the OCD group showed predominant global hypoactivation, particularly in the right superior temporal sulcus. Meanwhile, in the beta2 band CC, the MDD group showed predominant global hyperactivation, particularly in the right lingual gyrus. These region-specific differences were also reported in previous brain volumetric studies. The cortical volume of the right superior temporal gyrus (R23) was decreased in the patients with $O C D{ }^{26}$, and that of the right lingual gyrus (R11) was increased in the patients with MDD ${ }^{29}$. Our results showing the differences in local activation might be attributed to the brain morphological differences.

\section{Disease-specific patterns of PLV connectivity}

The lowered functional connectivity of theta band between the left lingual gyrus (R10) and the left hippocampus (R24) in the theta band DMN_was predominantly observed in the BD, SZ, PTSD, PD, and MDD groups. Hippocampal theta rhythm is associated with episodic memory ${ }^{30}$, and lingual gyrus is linked to encoding and retrieval of the visual memory ${ }^{25,31}$. Our results support prior studies that functional under-connectivity between lingual gyrus and hippocampus is related to the decline of cognitive function ${ }^{32,33}$, which are major pathophysiology of psychiatric disorder $25,34,35$.

There are some limitations in our study. First, some disease groups have relatively small numbers of participants. Second, the patients were not obtained in the drug naive state. Finally, symptomatic severity was not controlled.

In conclusion, we tried to compare the resting-state multiband EEG-based DMN for major psychiatric disorder groups, by patterning it to be visualized at a glance. Our results showed that EEG based DMN is a clinically useful and pathologically relevant method to evaluate major psychiatric disorders. Future work is needed to explore the relationship between DMN and symptomatic severity.

\section{Declarations}

\section{ACKNOWLEDGMENTS}


"This work was supported by the Brain Research Program through the National Research Foundation of Korea from the Ministry of Science, ICT \& Future Planning (NRF-2015M3C7A1028252) and the Korea Medical Device Development Fund grant funded by the Korea government (the Ministry of Science and ICT, the Ministry of Trade, Industry and Energy, the Ministry of Health \& Welfare, Republic of Korea, the Ministry of Food and Drug Safety) (Project Number: 202013B10) to Seung Hwan Lee."

\section{Conflict of interest}

The authors report no biomedical financial interests or potential conflicts of interest.

\section{References}

1. Raichle, M. E. The brain's default mode network. Annu. Rev. Neurosci.38, 433-447 (2015).

2. Mak, L. E. et al. The default mode network in healthy individuals: A systematic review and metaanalysis. Brain Connect.7, 25-33 (2017).

3. Smitha, K. A. et al. Resting state fMRI: A review on methods in resting state connectivity analysis and resting state networks. Neuroradiol. J.30, 305-317 (2017).

4. Hohenfeld, C., Werner, C. J. \& Reetz, K. Resting-state connectivity in neurodegenerative disorders: Is there potential for an imaging biomarker? Neurolmage Clin.18, 849-870 (2018).

5. van Son, D. et al. Frontal EEG theta/beta ratio during mind wandering episodes. Biol. Psychol.140, 1927 (2019).

6. Raichle, M. E. et al. A default mode of brain function. Proc. Natl. Acad. Sci. U. S. A.98, 676-682 (2001).

7. Garrity, A. G. et al. Abberant 'default mode' functional connectivity in schizophrenia. Am. J. Psychiatry164, 450-457 (2007).

8. Buckner, R. L., Andrews-Hanna, J. R. \& Schacter, D. L. The brain's default network: Anatomy, function, and relevance to disease. Ann. N. Y. Acad. Sci.1124, 1-38 (2008).

9. Sheline, Y. I. et al. The default mode network and self-referential processes in depression. Proc. Natl. Acad. Sci. U. S. A.106, 1942-1947 (2009).

10. Jerbi, K. et al. Exploring the electrophysiological correlates of the default-mode network with intracerebral EEG. Front. Syst. Neurosci.4, 27 (2010).

11. Forsyth, A. et al. Comparison of local spectral modulation, and temporal correlation, of simultaneously recorded EEG/fMRI signals during ketamine and midazolam sedation.

Psychopharmacology (Berl).235, 3479-3493 (2018). 
12. Smailovic, U. et al. Quantitative EEG power and synchronization correlate with Alzheimer's disease CSF biomarkers. Neurobiol. Aging63, 88-95 (2018).

13. Hsiao, F. J., Wang, Y. J., Yan, S. H., Chen, W. T. \& Lin, Y. Y. Altered oscillation and synchronization of default-mode network activity in mild Alzheimer's disease compared to mild cognitive impairment: An eectrophysiological study. PLoS One8, e68792 (2013).

14. Krukow, P., Jonak, K., Grochowski, C., Plechawska-Wójcik, M. \& Karakuła-Juchnowicz, H. Resting-state hyperconnectivity within the default mode network impedes the ability to initiate cognitive performance in first-episode schizophrenia patients. Prog. Neuro-Psychopharmacology Biol. Psychiatry102, 109959 (2020).

15. Cardoso, T., Bauer, I. E., Meyer, T. D., Kapczinski, F. \& Soares, J. C. Neuroprogression and cognitive functioning in bipolar disorder: A systematic review. Curr. Psychiatry Rep.17, 75 (2015).

16. Takahashi, S. Heterogeneity of schizophrenia: Genetic and symptomatic factors. Am. J. Med. Genet. Part B Neuropsychiatr. Genet.162, 648-652 (2013).

17. Price, R. B. et al. Parsing heterogeneity in the brain connectivity of depressed and healthy adults during positive mood. Biol. Psychiatry81, 347-357 (2017).

18. Guo, W. et al. Abnormal default-mode network homogeneity in first-episode, drug-naive major depressive disorder. PLoS One9, e91102 (2014).

19. Guo, W. et al. Abnormal default-mode network homogeneity in first-episode, drug-naive schizophrenia at rest. Prog. Neuro-Psychopharmacology Biol. Psychiatry49, 16-20 (2014).

20. Di Lorenzo, G. et al. Altered resting-state EEG source functional connectivity in schizophrenia: The effect of illness duration. Front. Hum. Neurosci.9, 234 (2015).

21. Horvath, A. et al. EEG and ERP biomarkers of Alzheimer's disease: A critical review. Front. Biosci. Landmark23, 183-220 (2018).

22. Creese, B. et al. Examining the association between genetic liability for schizophrenia and psychotic symptoms in Alzheimer's disease. Transl. Psychiatry9, 273 (2019).

23. van Driel, J., Knapen, T., van Es, D. M. \& Cohen, M. X. Interregional alpha-band synchrony supports temporal cross-modal integration. Neuroimage101, 404-415 (2014).

24. Shin, N. Y., Lee, T. Y., Kim, E. \& Kwon, J. S. Cognitive functioning in obsessive-compulsive disorder: A meta-analysis. Psychol. Med.44, 1121-1130 (2014).

25. Li, Y. et al. Abnormal resting-state functional connectivity strength in mild cognitive impairment and its conversion to Alzheimer's disease. Neural Plast.2016, 4680972 (2016). 
26. Moreira, P. S. et al. The neural correlates of obsessive-compulsive disorder: a multimodal perspective. Transl. Psychiatry7, e1224 (2017).

27. Hou, J. et al. Morphologic and functional connectivity alterations of corticostriatal and default mode network in treatment-naïve patients with obsessive-compulsive disorder. PLoS One8, e83931 (2013).

28. Brennan, B. P. et al. Lower posterior cingulate cortex glutathione levels in obsessive-compulsive disorder. Biol. Psychiatry Cogn. Neurosci. Neuroimaging1, 116-124 (2016).

29. Du, M. et al. Brain grey matter volume alterations in late-life depression. J. Psychiatry Neurosci.39, 397-406 (2014).

30. Heusser, A. C., Poeppel, D., Ezzyat, Y. \& Davachi, L. Episodic sequence memory is supported by a thetagamma phase code. Nat. Neurosci.19, 1374-1380 (2016).

31. Roland, P. E. \& Gulyás, B. Visual memory, visual imagery, and visual recognition of large field patterns by the human brain: functional anatomy by positron emission tomography. Cereb. Cortex5, 79-93 (1995).

32. Samudra, N. et al. Alterations in hippocampal connectivity across the psychosis dimension. Psychiatry Res. - Neuroimaging233, 148-157 (2015).

33. Kesler, S. R. et al. Altered resting state functional connectivity in young survivors of acute lymphoblastic leukemia. Pediatr. Blood Cancer61, 1295-1299 (2014).

34. Ferentinos, P. et al. Comorbidity of obsessive-compulsive disorder in bipolar spectrum disorders: Systematic review and meta-analysis of its prevalence. J. Affect. Disord.263, 193-208 (2020).

35. Lynch, D., Laws, K. R. \& McKenna, P. J. Cognitive behavioural therapy for major psychiatric disorder: Does it really work? A meta-analytical review of well-controlled trials. Psychol. Med.40, 9-24 (2010).

36. Tadel, F., Baillet, S., Mosher, J. C., Pantazis, D. \& Leahy, R. M. Brainstorm: A user-friendly application for MEG/EEG analysis. Comput. Intell. Neurosci.2011, 879716 (2011).

37. Gramfort, A., Papadopoulo, T., Olivi, E. \& Clerc, M. OpenMEEG: opensource software for quasistatic bioelectromagnetics. Biomed. Eng. Online9, 45 (2010).

38. Dasdemir, Y., Yildirim, E. \& Yildirim, S. Analysis of functional brain connections for positive-negative emotions using phase locking value. Cogn. Neurodyn.11, 487-500 (2017).

39. Rubinov, M. \& Sporns, O. Complex network measures of brain connectivity: Uses and interpretations. Neuroimage52, 1059-1069 (2010).

40. Curran, P. J., West, S. G. \& Finch, J. F. The robustness of test statistics to nonnormality and specification error in confirmatory factor analysis. Psychol. Methods1, 16-29 (1996). 
41. Kheirkhah, M. et al. The temporal and spatial dynamics of cortical emotion processing in different brain frequencies as assessed using the cluster-based permutation test: An MEG study. Brain Sci.10, 352 (2020).

\section{Figures}

a

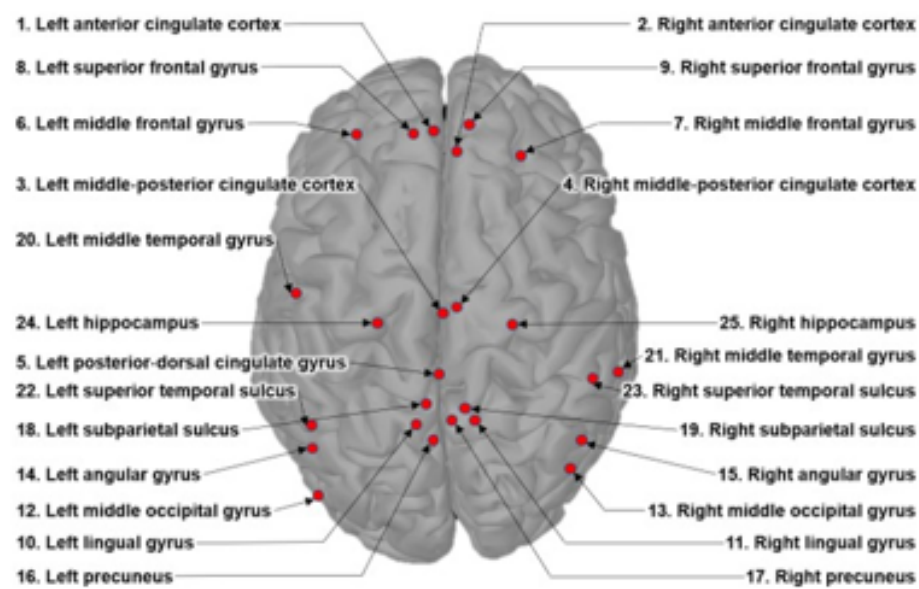

c

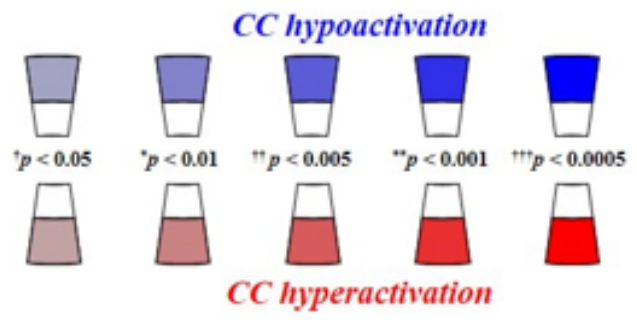

b

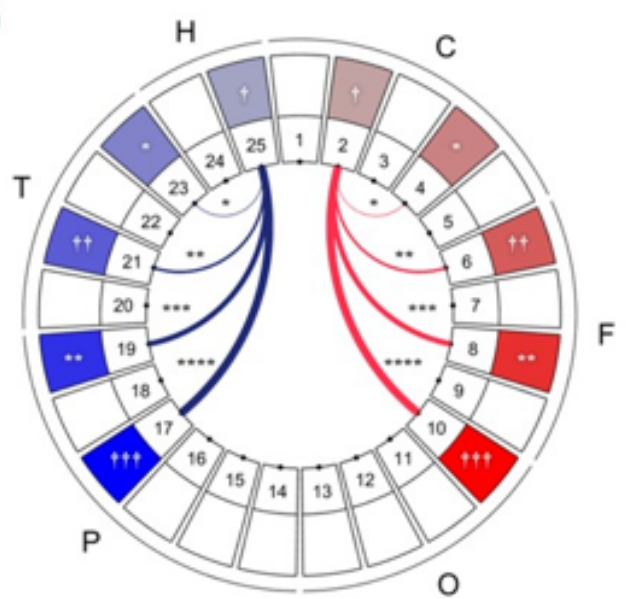

d

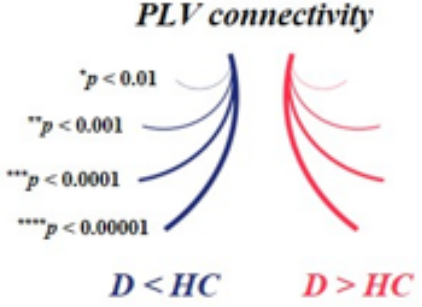

\section{Figure 1}

The ROls of the DMN and an exemplary DMN pattern with significance levels indicated. (a) $25 \mathrm{DMN}$ regions are displayed on the brain structure. (b) The visualized DMN pattern illustrates significantly different local CC activation and PLV connectivity of the disorder group compared with its demographically-matched HC group. In the visualization of both properties, red colors indicate the increased properties of the disease groups, while blue colors indicate the opposite case. The external capital letters denote brain regions (C: cingulate, F: frontal, O: occipital, P: parietal, T: temporal, $\mathrm{H}$ : hippocampal). (c) The significance levels of the $\mathrm{CC}$ activation are indicated by the color intensity with five levels. (d) The significance level of the PLV connectivity was indicated by the thickness of the curve with the logarithmic scale of the p-value. It is to be noted that only the PLV connectivities, the p-value of which is smaller than 0.01 , were illustrated in the pattern. For example in the pattern (b), right hippocampus (R25) and right precuneus (R17) showed strong PLV under-connectivity, and right anterior cingulate cortex (2) showed relatively weak but significant CC hyperactivation. It is noted that only the connectivities 
possessing significance levels $p<0.01$ were displayed. $+p<0.05$, $* p<0.01$, $+\uparrow p<0.005$, $* \star p<0.001$, ††† $p<0.0005, * \star \star p<0.0001, \star \star \star \star ~ p<0.00001$
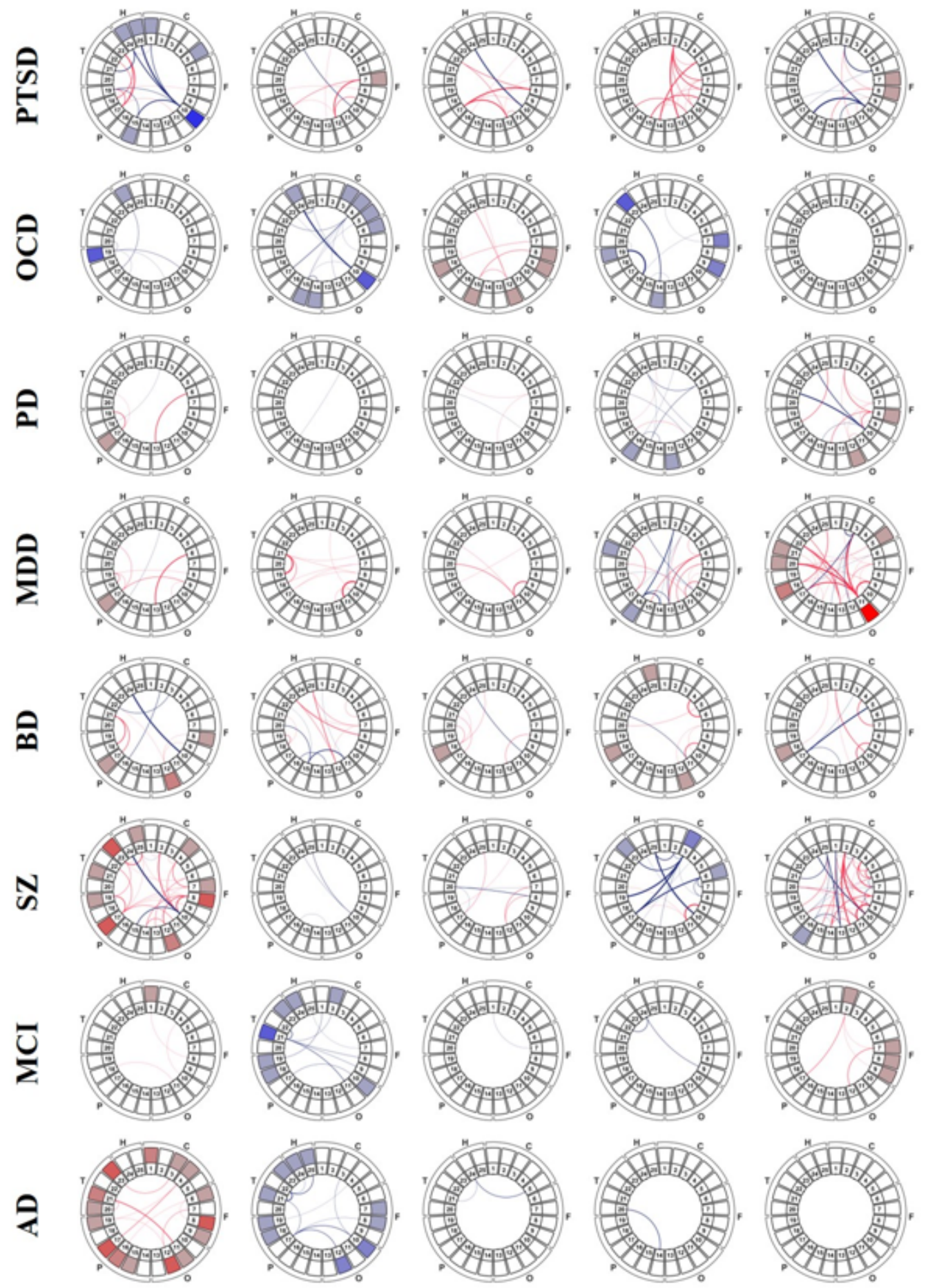

Theta

Alpha1

Alpha2

Beta1

Beta2

Figure 2

The DMN patterns at a glance in major psychiatric disorders for various frequency bands. The significance levels are illustrated in line with Fig. 1b. (PTSD: posttraumatic stress disorder, OCD: 
obsessive compulsive disorder, PD: panic disorder, MDD: major depressive disorder, BD: bipolar disorder, SZ: schizophrenia, MCl: mild cognitive impairment, AD: Alzheimer's disease)
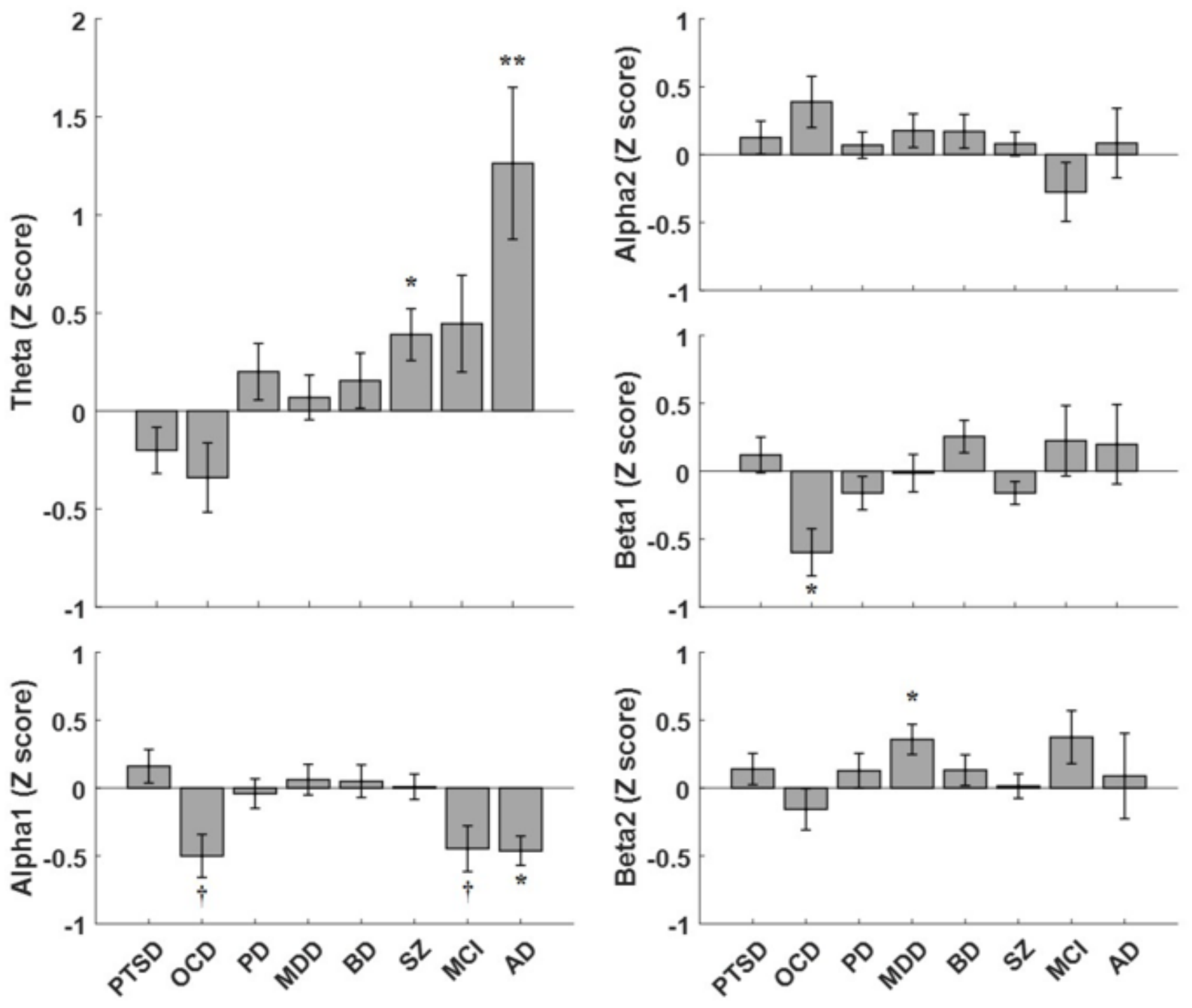

\section{Figure 3}

The global CC activation for various frequency bands. The global CC values of each group were normalized to those of the corresponding $\mathrm{HC}$ group, using z-transformation. Each bar indicates the averaged $z$-score of the global CC value of the disorder group. The error-bars indicate standard error. $+p<$ $0.07, * p<0.05, * \star p<0$. (PTSD: posttraumatic stress disorder, OCD: obsessive compulsive disorder, PD: panic disorder, MDD: major depressive disorder, BD: bipolar disorder, SZ: schizophrenia, MCl: mild cognitive impairment, AD: Alzheimer's disease) 


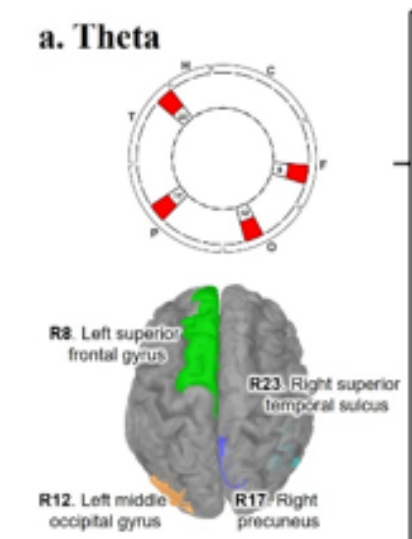

\section{c. Beta1}

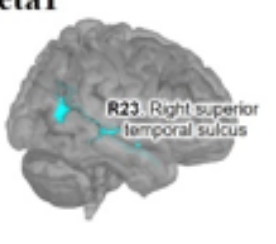

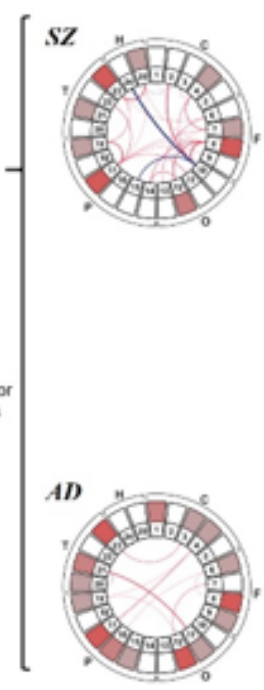
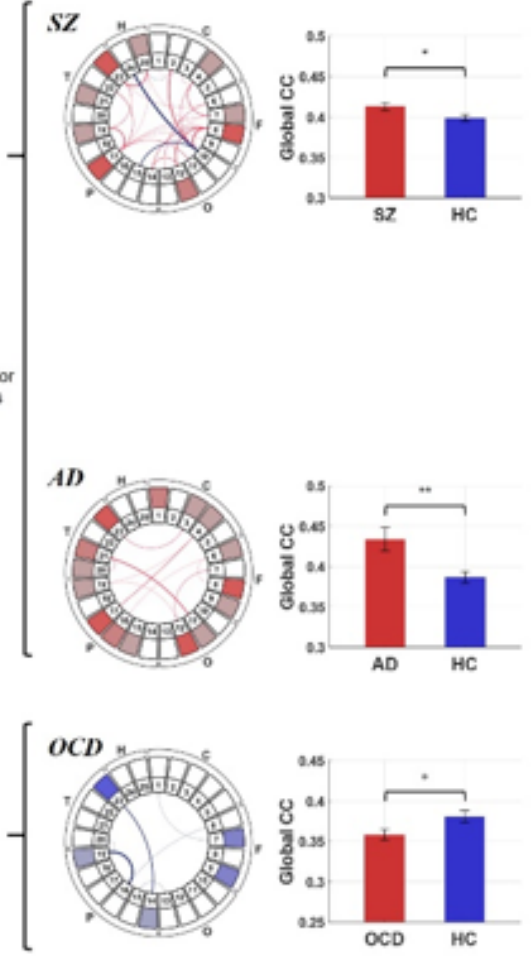

b. Alpha1

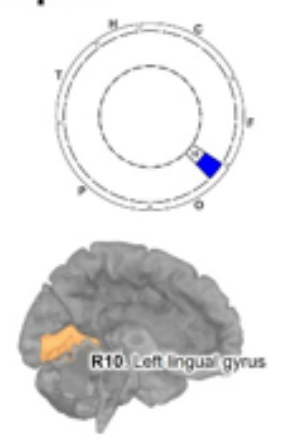

d. Beta2

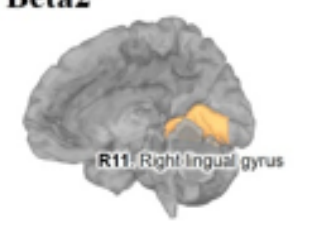

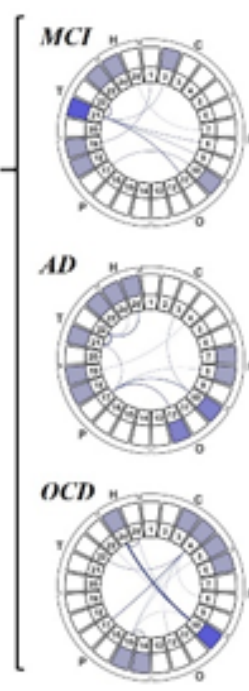
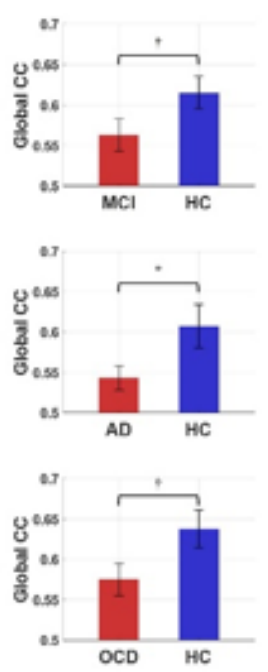

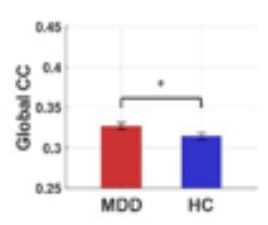

\section{Figure 4}

DMN CC activity analysis results. Only the results showing significantly different global activation are presented. For each frequency band, key ROls are highlighted on the brain image left side; additionally, if more than one is included, common key ROls are also illustrated as a form of DMN pattern. DMN patterns and the comparisons of global network activation (evaluated by global CCs) are presented right side. In the bar charts, red bars indicate disorder groups, and blue bars show HC groups. The error-bars indicate standard error. (a) In the theta band DMN, SZ and AD commonly showed strong hyperactivity in four regions: left superior frontal gyrus (R8), left middle occipital gyrus (R12), right precuneus (R17), and right superior temporal sulcus (R23). (b) In the alpha1 band DMN, MCl, OCD, and AD commonly showed hypoactivation in the left lingual gyrus (R10). (c) In the beta1 band DMN, OCD showed strong hypoactivation in the right superior temporal sulcus (R23). (d) In the beta2 band DMN, MDD showed strong hyperactivation in the right lingual gyrus (R11). $+p<0.07,{ }^{*} p<0.05$, $* * p<0.01$ (SZ: schizophrenia, AD: Alzheimer's disease, OCD: obsessive compulsive disorder, $\mathrm{MCl}$ : mild cognitive impairment, MDD: major depressive disorder, HC: healthy control) 


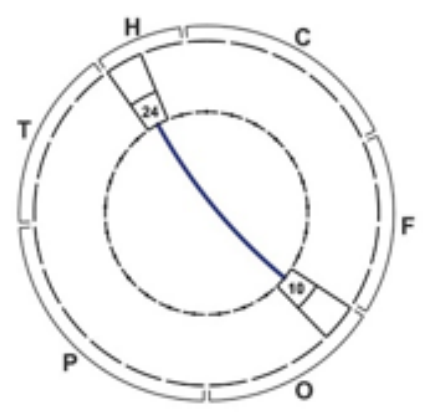

R10. Left lingual gyrus

R24. Left hippocampus
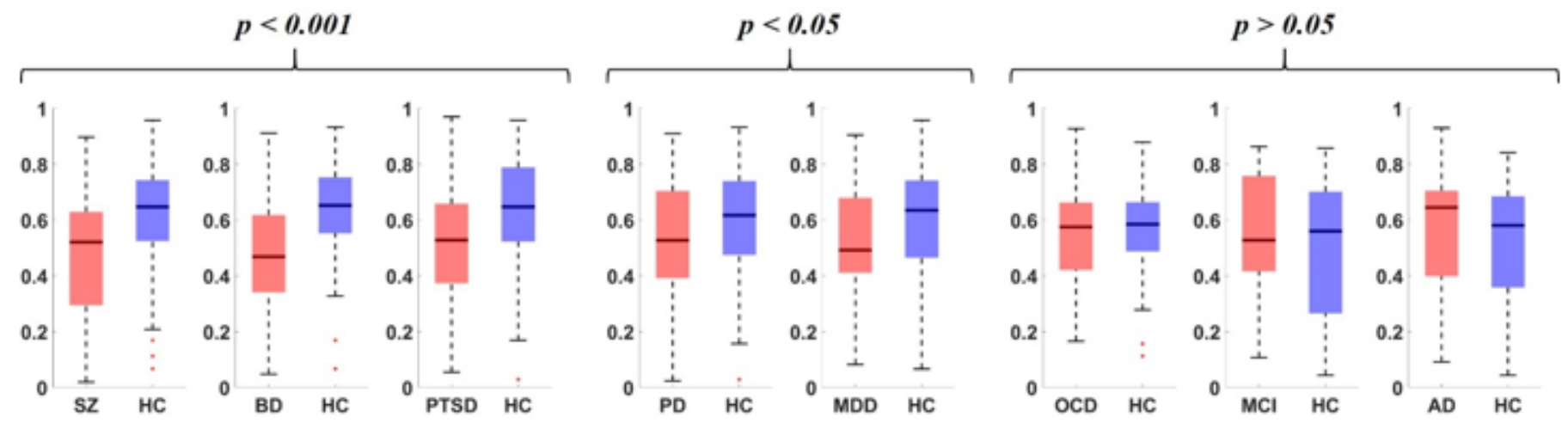

\section{Figure 5}

Comparison of theta band PLV connectivity between left lingual gyrus (R10) and left hippocampus (R24) for all disorder groups (red boxes) with their corresponding HC groups (blue boxes). The connectivity is illustrated as a form of DMN pattern (top left), which is highlighted on the brain image (top right). In the box plots (bottom), the black midlines indicate median values, the boxes indicate interquartile range (IQR), whiskers indicate the maximum and minimum value in the 1.5 times of IQR, and crosses (+) indicate outliers. The comparison results are displayed with three classes: the first class (SZ, BD, and PTSD) showed strongly decreased connectivity $(p<0.001)$; the second class (PD and MDD) showed relatively weak but significantly decreased connectivity $(p<0.05)$; and the third class $(O C D, M C l$, and $A D)$ showed no significantly different connectivity. (SZ: schizophrenia, BD: bipolar disorder, PTSD: posttraumatic stress disorder, $\mathrm{PD}$ : panic disorder, MDD: major depressive disorder, OCD: obsessive compulsive disorder, $\mathrm{MCl}$ : mild cognitive impairment, $\mathrm{AD}$ : Alzheimer's disease, $\mathrm{HC}$ : healthy control)

\section{Supplementary Files}

This is a list of supplementary files associated with this preprint. Click to download.

- Supplementary.docx 